Canadian University Music Review

Revue de musique des universités canadiennes

\title{
Marie-Thérèse Lefebvre. Serge Garant et la révolution \\ musicale au Québec. Biographie et écrits (1954-1984). \\ Montréal : Louise Courteau éditrice, 1986, 239 pages
}

\section{Lucie Paquin}

\section{Numéro 7, 1986}

URI : https://id.erudit.org/iderudit/1014084ar

DOI : https://doi.org/10.7202/1014084ar

Aller au sommaire du numéro

\section{Éditeur(s)}

Canadian University Music Society / Société de musique des universités canadiennes

\section{ISSN}

0710-0353 (imprimé)

2291-2436 (numérique)

Découvrir la revue

Citer ce compte rendu

Paquin, L. (1986). Compte rendu de [Marie-Thérèse Lefebvre. Serge Garant et la révolution musicale au Québec. Biographie et écrits (1954-1984). Montréal :

Louise Courteau éditrice, 1986, 239 pages]. Canadian University Music Review / Revue de musique des universités canadiennes, (7), 26-31.

https://doi.org/10.7202/1014084ar

All Rights Reserved (C Canadian University Music Society / Société de musique des universités canadiennes, 1986
Ce document est protégé par la loi sur le droit d'auteur. L'utilisation des services d'Érudit (y compris la reproduction) est assujettie à sa politique d'utilisation que vous pouvez consulter en ligne.

https://apropos.erudit.org/fr/usagers/politique-dutilisation/ 
MARIE-THERESE LEFEBVRE. Serge Garant et la révolution musicale au Québec. Biographie et écrits (1954-1984). Montréal : Louise Courteau éditrice, 1986, 239 pages.

La littérature musicologique a, jusqu'à ce jour, peu rendu compte de l'avènement de la modernité musicale au Québec, et c'est en vain que l'on chercherait un ouvrage analogue à celui présenté ici. ${ }^{1}$ L'étude exhaustive d'un tel phénomène nécessite une mise en parallèle de nombreux facteurs et ne peut certes se limiter à l'instigation d'un seul homme. Néanmoins, force nous est de constater le rôle déterminant et de tout premier ordre qu'a joué Serge Garant dans l'évolution et la diffusion de la musique contemporaine au Québec. Que ce soit à titre de critique, d'animateur, de conférencier, de chef d'orchestre, de compositeur ou de pédagogue, son engagement dans le monde musical fut sans défaillance et ce, pendant plus de trente ans. De ce point de vue, le livre de Marie-Thérèse Lefebvre, réunissant près de cent quarante textes écrits par Serge Garant entre 1954 et 1984 (textes auxquels deux autres, datés de 1986, se sont ajoutés), prend aujourd'hui une importance privilégiée puisque, à travers ce témoignage, "c'est l'histoire d'une société, de son émergence culturelle et de la démarche de son identité qui nous est racontée" (p.13).

Afin de bien situer les écrits de Garant dans une perspective socioculturelle, l'auteure consacre les soixante-dix premières pages du livre à une biographie succinte, ainsi qu'à une mise en place du contexte musical ayant prévalu entre les années 1920 et 1950, années où se sont infiltrées discrètement à Montréal les premières manifestations d'une modernité musicale.

1 Marie-Thérèse Lefebvre est aussi l'auteure de "La modernité dans la création musicale", cf. Lamonde et Trépanier (1986). 
A l'examen de ce profil historique, Marie-Thérèse Lefebvre constate l'aspect révolutionnaire, sans précédent dans l'histoire musicale canadienne, de la démarche créatrice de Serge Garant. Cet état de fait s'avère fondamental puisque, d'une part, il situe la position nonéquivoque de l'auteure - position d'ailleurs suggérée par le titre de l'ouvrage - et, d'autre part, il tend à remettre en question la convention selon laquelle "on ait souvent relié les débuts de cette modernité aux événements qui ont entouré le manifeste Refus global, à cause de la présence du compositeur Pierre Mercure parmi le groupe contestataire" (p.21). Les données historiques justifient en quelque sorte l'avant- gardisme de Garant, inspiré par les principes du sérialisme à une époque où Varèse, Webern, Schoenberg étaient encore inconnus au Canada.

Bien que brève, cette première partie est essentielle à la compréhension des écrits de Garant. L'on appréciera, en outre, le souci d'objectivité de l'auteure face à un sujet n'offrant point l'avantage sécurisant du recul historique. De nombreuses citations soutiennent de façon pertinente son point de vue. Un appendice joint également un complément d'informations : liste des concerts de Serge Garant à Sherbrooke, le programme des concerts de la Semaine internationale de musique actuelle (Montréal, 1961), ainsi qu'une liste chronologique détaillée des oeuvre du compositeur.

Dans une seconde partie, constituant à elle seule les deux-tiers du volume, Marie-Thérèse Lefebvre cède la parole au témoignage vivant de Serge Garant. L'intérêt et l'originalité de cette anthologie résident dans la diversité et l'organisation des textes choisis. L'itinéraire proposé s'articule en cinq thèmes : S'engager, Bousculer, Informer, Animer, Créer, et met en lumière divers aspects de la carrière de Garant. Les textes, brièvement commentés au préalable par l'auteure, proviennent de plusieurs sources : entrevues, conférences, discours, critiques, émissions radiophoniques et notes de programme. 
De façon générale, l'état d'esprit de ces textes s'inscrit sous le signe de la dénonciation, de la polémique et de la revendication. Plusieurs d'entre eux, à caractère journalistique, naissent de la plume d'un pamphlétaire. Ardent défenseur de ses convictions esthétiques, Garant ne craindra pas d'attaquer ouvertement critiques, compositeurs et interprètes et d'adopter, lorsqu'il le faut, un ton violent, voire même lapidaire, souvent relevé d'une touche habile d'humour cynique.

A une époque que nous qualifions aujourd'hui de "grande noirceur" les propos acerbes et pessimistes que tenait Serge Garant suscitèrent sans doute beaucoup de controverses. Un article de 1959, "Le compositeur moderne : paria de la musique" (p.105-106), résume à lui seul ce climat d'hostilité. Nous en retiendrons quelques extraits :

On le voit : de quelque côté qu'il se tourne, le jeune compositeur se trouve devant un mur. L'interprète n'est pas intéressé, le chef d'orchestre, forcé de plaire à la fois au réalisateur ou au comité d'orchestre, à ses musiciens et au public, n'ose pas imposer - le plus souvent en est incapable - une oeuvre nouvelle, à moins qu'elle ne soit très confortable. La critique, inutile d'en parler : elle est réservée aux oeuvrettes destinées aux couvents.

Le compositeur a le choix : s'intégrer à la "music business" ou écrire pour lui seul.

Et Garant conclut ainsi :

Nous avons besoin de quelques révoltés qui n'hésitent pas à tout casser, qui vomissent la stagnation et le culte des idoles qu'on nomme "le respect des traditions", et qui imposent, plutôt que l'amour pleurnicheur du passé, l'amour de la matière vivante.

La musique vit et vivra, sans doute. Mais il est bien dommage que ses plus féroces ennemis soient ceux-là mêmes qui s'en nourrissent : les musiciens.

Ces propos sont loin d'être gratuits et sous-tendent un commun dénominateur. Comme le mentionne Udo Kasemets (1979 : 119), 
le sujet traité est, avec quelques variantes, toujours le même : "s'il doit y avoir une culture musicale au Québec, ou au Canada d'ailleurs, elle doit prendre pour modèles les exemples et les critères établis par ce qu'il y a de meilleur au monde. La médiocrité, le provincialisme et l'esprit de clocher n'ont pas de place dans les salles de concert d'une nation en pleine croissance."

De plus, quel que soit le sujet abordé dans les écrits, le point de vue dominant demeure celui d'un compositeur. Contemporain de Boulez et de Stockhausen - "les chefs de file" - fortement inspiré par les recherches de Webern et de Varèse - "les deux seuls compositeurs de cette génération dont l'esprit est entièrement, et sans aucune nostalgie, moderne" (p.134) -, inquiet face à la nouvelle vague de romantisme et de "retours à" - "nous vivons maintenant dans un monde qui s'est laissé séduire par la facilité [...] quand on est rétro, c'est qu'on n'a rien à dire" (p.239) - Serge Garant n'a eu de cesse de s'interroger sur les principes fondamentaux de la composition musicale.

A cet égard, plusieurs de ses réflexions, commentaires ou analyses d'oeuvres contemporaines sont éloquents. L'on notera, à la lecture de ceux-ci, l'honnêteté, la lucidité et l'érudition de Serge Garant face au répertoire du XXe siècle. Son arrogance envers toute forme de traditionnalisme est manifeste et ne fait place à aucun compromis. En dépit de cette rigueur et de cette intransigeance, le geste créateur de Garant fut constamment tenaillé par une multitude de doutes et de remises en question, davantage peut-être ces dernières années où une seule pièce, Plages (1981), s'est ajoutée à son oeuvre :

Je cherche une langage nouveau et il ne m'intéresse pas de me promener dans ma bibliothèque et de me citer. [...] Il me semble que le geste même du créateur est de renouveler son langage de l'intérieur. Je ne veux pas que mon langage ne soit qu'une succession de "trucs" d'écriture, car il est très facile de s'en faire un répertoire. (p.238) 
S'il est trop tôt pour prévoir ce que l'histoire retiendra de l'oeuvre de Serge Garant, nombre de ses écrits parviendront du moins à supprimer quelques préjugés entretenus jusqu'à aujourd'hui sur l'esthétique du compositeur, laquelle fut souvent qualifiée d"intello-structuraliste" , laissant pour compte toute émotivité et sensibilité. A cet effet, soulignons une entrevue réalisée en juillet $1986^{2}$ dans laquelle Garant expose de façon fort explicite sa conception du temps musical, l'influence de l'art pictural sur son oeuvre, ainsi que sa préoccupation essentielle qui consiste à "rechercher d'abord un équilibre entre l'intuition, l'intelligence, la technique, la sensibilité et faire en sorte que la poésie se niche au creux d'une réussite technique" (p.239).

Voilà donc un ouvrage qui, en raison de la diversité des sujets qu'il aborde, comble de toute évidence une grande lacune : mais là n'est pas son seul mérite. Les écrits de Garant, loin de tomber dans le narcissisme et la complaisance, sont le témoin éloquent d'une démarche créatrice résolument moderne, authentique et passionnée, mais aussi de cette aventure périlleuse dans laquelle s'est engagé le Québec depuis les années 50. De plus, cette anthologie fournit un éclairage nouveau sur notre présent musical, encore incertain mais, à tout le moins, prometteur.

Une seule réserve, bien qu'elle soit inhérente à ce genre d'ouvrage : les nombreuses redites, particulièrement en ce qui concerne Webern. Nous pouvons toutefois comprendre que celles-ci répondent à un souci d'exhaustivité de la part de l'auteure, et les propos de Garant lui-même tempèrent quelque peu notre reproche : "Pour arriver à implanter cette idée absolument essentielle que la musique, c'est un organisme vivant, que ce n'est pas un art de musée, il fallait absolument continuer malgré tout à dire et à redire et à répéter toujours les mêmes choses. Pendant des années, je n'ai parlé que de Webern" (p.75).

2 On trouvera un fragment de cette entrevue à la suite du présent compte rendu. (N.d.E.) 
Certes, le regard que porte Serge Garant sur la vie musicale depuis l'après-guerre ne nous offre qu'une interprétation parmi d'autres, mais il saura, nous l'espérons, stimuler les chercheurs à étudier cette période foisonnante de notre histoire.

\section{Lucie Paquin}

\section{RÉFÉRENCES}

KASEMETS, U.

1977: "Serge Garant", Compositeurs canadiens contemporains. Montréal : Presses de l'Université du Québec, 117-121.

LAMONDE, Y. et E. TRÉPANIER.

1986: L'avènement de la modernité culturelle au Québec. Institut québécois de recherche sur la culture, 1986. 